ORNL/TM-12852

\section{OAK RIDGE}

NATIONAL

LABORATORY

MARTIN MARIETMA

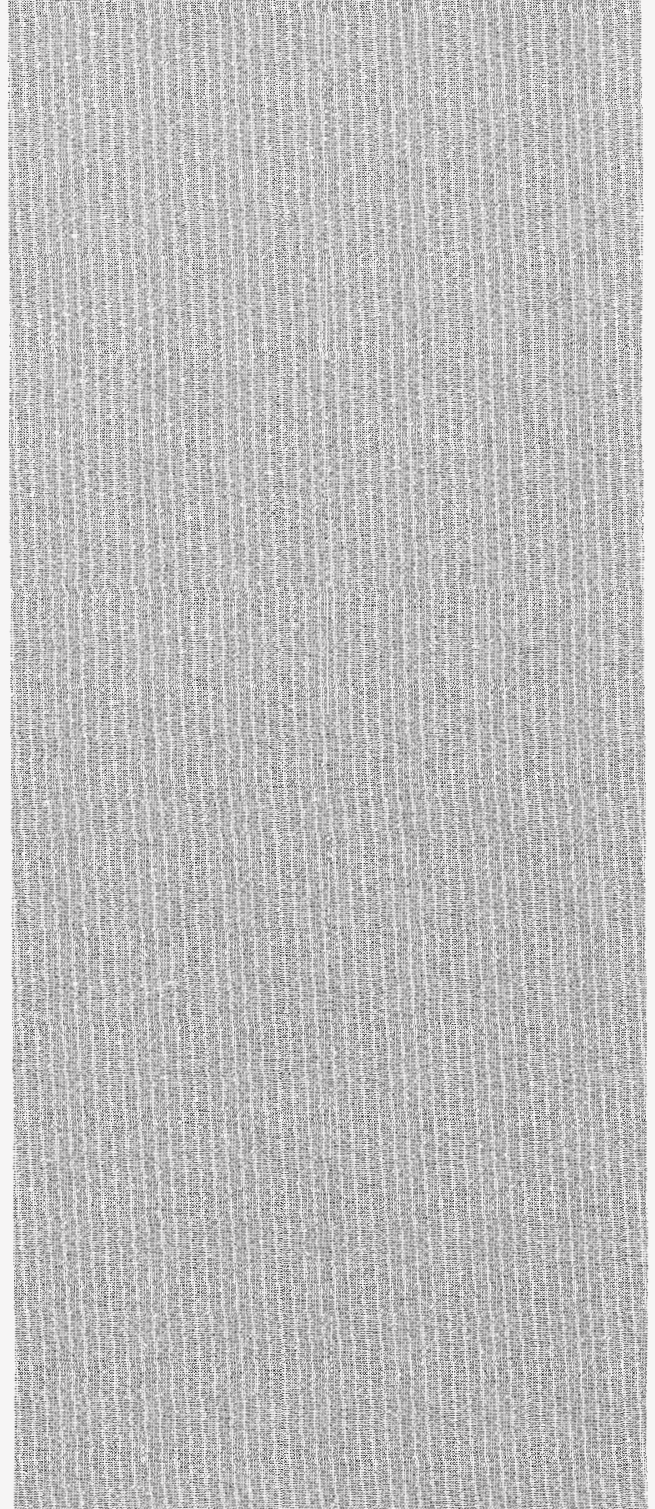

MANAGED BY

MARTIN MARIETTA ENERGY SYSTEMS, INC.

FOR THE UNIED STATES

DEPARTMENT OF ENERGY

\section{A Note on the Total Least Squares Problem for Coplanar Points}

Steven L. Lee 
This report has been reproduced directly from the best available copy.

Available to DOE and DOE contractors from the Office of Scientific and Technical Information, P.O. Box 62, Oak Ridge, TN 37831; prices available from (615) 576-8401, FTS 626-8401.

Available to the public from the National Technical Information Service, U.S. Department of Commerce, 5285 Port Royal Rd., Springfield, VA 22161.

This report was prepared as an account of work sponsored by an agency of the United States Government. Neither the United States Government nor any agency thereof, nor any of their employees, makes any warranty, express or implied, or assumes any legal liability or responsibility for the accuracy, completeness, or usefulness of any information, apparatus, product, or process disclosed, or represents that its use would not infringe privately owned rights. Reference herein to any specific commercial product, process, or service by trade name, trademark, manufacturer, or otherwise, does not necessarily constitute or imply its endorsement, recommendation, or favoring by the United States Government or any agency thereof. The views and opinions of authors expressed herein do not necessarily state or reflect those of the United States Government or any agency thereof. 


\section{DISCLAIMER}

Portions of this document may be illegible in electronic image products. Images are produced from the best available original document. 
ORNL/TM-12852

Engineering Physics and Mathematics. Division

\title{
A NOTE ON THE TOTAL LEAST SQUARES PROBLEM FOR COPLANAR POINTS
}

\author{
Steven L. Lee
}

\begin{abstract}
Mathematical Sciences Section Oak Ridge National Laboratory

P.O. Box 2008, Bldg. 6012

Oak Ridge, TN 37831-6367

slee@msr.epm.ornl.gov
\end{abstract}

Date Published: September 1994

Research was supported by the Applied Mathematical Sciences Research Program of the Office of Energy Research, U.S. Department of Energy.

Prepared by the

Oak Ridge National Laboratory

Oak Ridge, Tennessee 37831

managed by

Martin Marietta Energy Systems, Inc.

for the

U.S. DEPARTMENT OF ENERGY

under Contract No. DE-AC05-84OR21400

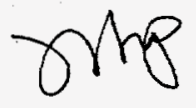





\section{Contents}

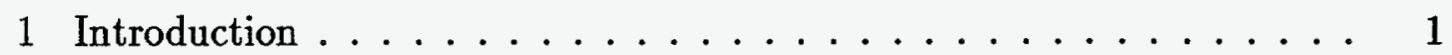

2 Main results $\ldots \ldots \ldots \ldots \ldots \ldots \ldots \ldots \ldots \ldots \ldots$

3 References ..................... 6 
.

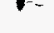

' 


\title{
A NOTE ON THE TOTAL LEAST SQUARES PROBLEM FOR COPLANAR POINTS
}

\author{
Steven L. Lee
}

\begin{abstract}
The Total Least Squares (TLS) fit to the points $\left(x_{k}, y_{k}\right), k=1, \cdots, n$, minimizes the sum of the squares of the perpendicular distances from the points to the line. This sum is the TLS error, and minimizing its magnitude is appropriate if $x_{k}$ and $y_{k}$ are uncertain. A priori formulas for the TLS fit and TLS error to coplanar points were originally derived by Pearson in [Phil. Mag., 2 (1901), pp. 559-572], and they are expressed in terms of the mean, standard deviation and correlation coefficient of the data. In this note, these TLS formulas are derived in a more elementary fashion. The TLS fit is obtained via the ordinary least squares problem and the algebraic properties of complex numbers. The TLS error is formulated in terms of the triangle inequality for complex numbers.
\end{abstract}




\section{Introduction}

Given the set of points $\left(x_{k}, y_{k}\right), k=1, \cdots, n$, it is often desirable to find the straight line

$$
y=\alpha x+\beta
$$

that minimizes the sum of the squares of the perpendicular distances from the points to the line. Properly speaking, the above is a total least squares (TLS) problem. The desired line is the TLS fit to the points $\left(x_{k}, y_{k}\right)$, and the TLS error is the sum to be minimized. Such a fitting is appropriate if $x_{k}$ and $y_{k}$ are subject to error. For some additional discussion of this topic, see the recent paper by Nievergelt [4], and the references therein. An analysis of this problem, and more general TLS problems, is also given by Golub and Van Loan in [1] and by Van Huffel and Vanderwalle in [2].

\section{Main results}

In the least squares (LS) problem, we are interested in finding the straight line $y=a x+b$ that minimizes the sum of the squares of the vertical distances from the points $\left(x_{k}, y_{k}\right)$ to the line. It is well known that the solution to the LS problem can be obtained by solving the following system of "normal equations":

$$
\left[\begin{array}{cc}
n & \sum x_{k} \\
\sum x_{k} & \sum x_{k}^{2}
\end{array}\right]\left[\begin{array}{l}
b \\
a
\end{array}\right]=\left[\begin{array}{l}
\sum y_{k} \\
\sum x_{k} y_{k}
\end{array}\right] .
$$

The solution is unique, unless $x_{1}=\cdots=x_{n}$ (i.e., the points are vertically aligned).

We can learn a great deal about the TLS problem by first transforming it into a LS problem and then applying the normal equations (2). A few observations are needed before pursuing this approach. In particular, for the mean values

$$
\bar{x}=\frac{1}{n} \sum x_{k} \quad \text { and } \quad \bar{y}=\frac{1}{n} \sum y_{k},
$$

let

$$
\tilde{x}_{k}=x_{k}-\bar{x} \quad \text { and } \quad \tilde{y}_{k}=y_{k}-\bar{y}
$$

so that the TLS fit to the points $\left(\tilde{x}_{k}, \tilde{y}_{k}\right)$ passes through the origin [4, pg. 259]. 
The TLS slope $\alpha$ and TLS error are unaffected by this translation. We now proceed to work in terms of $\left(\tilde{x}_{k}, \tilde{y}_{k}\right)$ to determine the slope and angle at which the TLS fit crosses the origin. For notational convenience, let's denote these centered points as the complex numbers

$$
\tilde{z}_{k}=\tilde{x}_{k}+i \tilde{y}_{k}
$$

and let

$$
0 \leq \tau<\pi
$$

denote the angle (in the counterclockwise direction) that the TLS fit makes with the positive real axis. Thus, we have the trivial relations that slope $\alpha=\tan (\tau)$ and $\tau=\tan ^{-1}(\alpha)$. The analysis that follows is simpler if we work in terms of $\tau$.

In principle, we can transform the TLS problem into a LS problem by rotating the points $\tilde{z}_{k}$ by $-\tau$ so that the TLS fit to the points

$$
w_{k}=e^{-i \tau} \tilde{z}_{k}
$$

lies along the real axis. The TLS error to the points $\tilde{z}_{k}$ is unaffected by this rotation, and the perpendicular distances from the points $w_{k}$ to the TLS fit lie strictly in the vertical direction. In this way, we establish the real axis as the solution to a TLS and LS problem.

Although the $\tau$ in (7) is unknown, we can determine some of its basic properties by applying the normal equations (2) to the points $w_{k}$; namely,

$$
\left[\begin{array}{cc}
n & \sum \operatorname{Re}\left(w_{k}\right) \\
\sum \operatorname{Re}\left(w_{k}\right) & \sum \operatorname{Re}^{2}\left(w_{k}\right)
\end{array}\right]\left[\begin{array}{l}
b \\
a
\end{array}\right]=\left[\begin{array}{l}
\sum \operatorname{Im}\left(w_{k}\right) \\
\sum \operatorname{Re}\left(w_{k}\right) \operatorname{Im}\left(w_{k}\right)
\end{array}\right] .
$$

Equation (8) has the solution $a=0, b=0$ since the real axis is the LS fit to the points (7). Based on this unique LS solution, the right-hand side of (8) must also be zero, and we find that the unknown $\tau$ satisfies

$$
\sum \operatorname{Im}\left(e^{-i \tau} \tilde{z}_{k}\right)=0
$$

and

$$
\sum \operatorname{Re}\left(e^{-i \tau} \tilde{z}_{k}\right) \operatorname{Im}\left(e^{-i \tau} \tilde{z}_{k}\right)=0 .
$$

The first condition (9) says that the sum of the perpendiculars, $d_{k}$, is zero. The 
second condition (10) is also valuable since the summation of cross terms suggests a strategy for explicitly determining $\tau$. Later, we show that we can obtain the total least squares error,

$$
\text { TLS error } \equiv \sum\left|d_{k}\right|^{2}=\sum \operatorname{Im}^{2}\left(e^{-i \tau} \tilde{z}_{k}\right)
$$

via a formula that does not involve $\tau$.

To deduce the TLS angle $\tau$, we begin by expanding the term

$$
\begin{aligned}
\rho & =\sum\left(e^{-i \tau} \tilde{z}_{k}\right)^{2} \\
& =\sum\left[\operatorname{Re}\left(e^{-i \tau} \tilde{z}_{k}\right)+i \operatorname{Im}\left(e^{-i \tau} \tilde{z}_{k}\right)\right]^{2} \\
& =\sum \operatorname{Re}^{2}\left(e^{-i \tau} \tilde{z}_{k}\right)-\sum \operatorname{Im}^{2}\left(e^{-i \tau} \tilde{z}_{k}\right)+2 i\left\{\sum \operatorname{Re}\left(e^{-i \tau} \tilde{z}_{k}\right) \operatorname{Im}\left(e^{-i \tau} \tilde{z}_{k}\right)\right\}
\end{aligned}
$$

Notice that the summation of cross terms, in curly brackets, is zero due to condition (10). Thus, the third important property of the unknown $\tau$ is that $\rho$ is a real number.

To proceed further, we now compute the complex number

$$
s=\sum \tilde{z}_{k}^{2}
$$

and simplify the term

$$
\rho=\sum\left(e^{-i \tau} \tilde{z}_{k}\right)^{2}=e^{-2 i \tau} \sum \tilde{z}_{k}^{2}=e^{-2 i \tau} s
$$

to get the exponential form

$$
s=\rho e^{2 i \tau} .
$$

We can establish $\rho$ as a positive real number by denoting $0 \leq 2 \tau<2 \pi$ as the angle (in the counterclockwise direction) that $s$ makes with the positive real axis. By working with the complex form of $s$, we can exhibit the real and imaginary parts via

$$
s=\sum \tilde{z}_{k}^{2}=\sum\left(\tilde{x}_{k}+i \tilde{y}_{k}\right)^{2}=\sum \tilde{x}_{k}^{2}-\sum \tilde{y}_{k}^{2}+2 i \sum \tilde{x}_{k} \tilde{y}_{k}
$$

and, for the angle in the exponential form, determine that

$$
\tan (2 \tau)=\frac{\operatorname{Im}(s)}{\operatorname{Re}(s)}=\frac{2 \sum \tilde{x}_{k} \tilde{y}_{k}}{\sum \tilde{x}_{k}^{2}-\sum \tilde{y}_{k}^{2}} .
$$


In general, there are two values of $\tau$ that satisfy (19); these two values will differ by $\pi / 2$. The TLS angle is the one that also satisfies (9). Other approaches to deriving (19) appear in [5] and [3, Chapter 13, Section 4]. If $\tan (\tau)$ is defined, we then have the TLS slope $\alpha=\tan (\tau)$. The point-slope form

$$
y-\bar{y}=\alpha(x-\bar{x})
$$

can be used to obtain the $\beta$ term of the TLS fit (1).

An a priori formula for the TLS error comes from

$$
\sum\left|e^{-i \tau} \tilde{z}_{k}\right|^{2}=\sum\left|\tilde{z}_{k}\right|^{2}
$$

and the following lemma.

Lemma 2.1. For the total least squares angle $\tau$ to the points $\tilde{z}_{k}$,

$$
\sum\left(e^{-i \tau} \tilde{z}_{k}\right)^{2}=\left|\sum \tilde{z}_{k}^{2}\right|
$$

Proof: From (12), we have

$$
\sum\left(e^{-i \tau} \tilde{z}_{k}\right)^{2}=\rho
$$

From the exponential form of $s$ in (17), we know that $\rho$ equals the magnitude of $s$. Thus, we finish with

$$
\rho=|s|=\left|\sum \tilde{z}_{k}^{2}\right|
$$

For the TLS angle $\tau$, we can also show that

$$
\sum\left|e^{-i \tau} \tilde{z}_{k}\right|^{2}=\sum \operatorname{Re}^{2}\left(e^{-i \tau} \tilde{z}_{k}\right)+\sum \operatorname{Im}^{2}\left(e^{-i \tau} \tilde{z}_{k}\right)
$$

and, from (12)-(14),

$$
\sum\left(e^{-i \tau} \tilde{z}_{k}\right)^{2}=\sum \operatorname{Re}^{2}\left(e^{-i \tau} \tilde{z}_{k}\right)-\sum \operatorname{Im}^{2}\left(e^{-i \tau} \tilde{z}_{k}\right)
$$


At this stage, notice that (25) and (26) differ by

$$
2 \sum \operatorname{Im}^{2}\left(e^{-i \tau} \tilde{z}_{k}\right)
$$

which is precisely twice the TLS error for the problem; see (11). Moreover, (21) and (22) show that this difference is a readily computable quantity; namely,

$$
2(\text { TLS error })=\sum\left|\tilde{z}_{k}\right|^{2}-\left|\sum \tilde{z}_{k}^{2}\right|
$$

Theorem 2.2. Given the points $\left(x_{k}, y_{k}\right), k=1, \cdots, n$, let

$$
z_{k}=x_{k}+i y_{k} \quad \text { and } \quad \bar{z}=\frac{1}{n} \sum z_{k}
$$

so that

$$
\tilde{z}_{k}=z_{k}-\bar{z}
$$

The error for the total least squares fit is

$$
\sum\left|d_{k}\right|^{2}=\frac{1}{2}\left(\sum\left|\tilde{z}_{k}\right|^{2}-\left|\sum \tilde{z}_{k}^{2}\right|\right)
$$

where $\left|d_{k}\right|$ is the perpendicular distance from $\left(x_{k}, y_{k}\right)$ to the fit.

Proof: The theorem follows from (28).

The TLS error formula in (31) is a concise version of Pearson's formula [5, pg. 566]. For comparison, note that the latter result reduces to

$$
\sum\left|d_{k}\right|^{2}=\frac{1}{2}\left(\sum \tilde{x}_{k}^{2}+\sum \tilde{y}_{k}^{2}-\left[\left(\sum \tilde{x}_{k}^{2}-\sum \tilde{y}_{k}^{2}\right)^{2}+4\left(\sum \tilde{x}_{k} \tilde{y}_{k}\right)^{2}\right]^{1 / 2}\right)
$$

Moreover, by arranging the formula (31) as

$$
\left|\sum \tilde{z}_{k}^{2}\right|+2 \sum\left|d_{k}\right|^{2}=\sum\left|\tilde{z}_{k}\right|^{2}
$$

we can drop the term $2 \sum\left|d_{k}\right|^{2}$ and thereby obtain the triangle inequality

$$
\left|\sum \tilde{z}_{k}^{2}\right| \leq \sum\left|\tilde{z}_{k}^{2}\right|=\sum\left|\tilde{z}_{k}\right|^{2}
$$

as a special case. Thus, (33) establishes an important relation between the TLS error to $z_{k}$ and the triangle inequality for $\tilde{z}_{k}^{2}$. In particular, we find that the error 
in the TLS fit equals half the "error" in the triangle inequality for the square of the centered points, $\tilde{z}_{k}^{2}$ (see Theorem 2.2).

Unfortunately, this new derivation for the TLS fit and TLS error does not generalize to higher dimensions. Algorithms for fitting a hyperplane to higherdimensional data are described in $[1,4]$.

\section{A.cknowledgments}

I wish to thank Gene Golub for helpful pointers to literature concerning the total least squares problem.

\section{References}

[1] Gene H. Golub and Charles F. Van Loan. An analysis of the total least squares problem. SIAM J. Numer. Anal., 17:883-893, 1980.

[2] Sabine Van Huffel and Joos Vanderwalle. The Total Least Squares Problem: Computational Aspects and Analysis. Society for Industrial and Applied Mathematics, Philadelphia, PA, 1991.

[3] I. Linnik. Method of Least Squares and Principles of the Theory of Observations. Pergamon Press, New York, 1961.

[4] Yves Nievergelt. Total least squares: State-of-the-art regression in numerical analysis. SIAM Review, 36(2):258-264, June 1994.

[5] Karl Pearson. On lines and planes of closest fit to points in space. Phil. Mag., 2:559-572, 1901. 
ORNL/TM-12852

\section{INTERNAL DISTRIBUTION}

1. B.R. Appleton

2. T.S. Darland

3. E.F. D'Azevedo

4. J.M. Donato

5. J.J. Dongarra

6. J.B. Drake

7. G.A. Geist

8-12. M.R. Leuze

13. N.M. Nachtigal

14-18. E.G. $\mathrm{Ng}$

19. C.E. Oliver

20. P.M. Papadopoulos
21-25. S.A. Raby

26. C.H. Romine

27. T.H. Rowan

28. B.D. Semeraro

29-33. R.F. Sincovec

34-38. R.C. Ward

39. Central Research Library

40. ORNL Patent Office

41. K-25 Appl Tech Library

42. Y-12 Technical Library

43. Lab Records Dept - RC

44-45. Laboratory Records Dept

\section{EXTERNAL DISTRIBUTION}

46. Loyce Adams, Dept. of Applied Mathematics, FS-20, University of Washington, Seattle, WA 98195

47. Fernando Alvarado, University of Wisconsin, 1425 Johnson Drive, Madison, WI 53706-1607

48. Greg Ammar, Dept. of Mathematical Sciences, Northern Illinois University, DeKalb, IL 60115

49. Mario Arioli, IAN-CNR, c/o Dipartimento di Matematica, 209, via Abbiategrasso, 27100 Pavia, Italy

50. Steven Ashby, Lawrence Livermore National Lab, P.O. Box 808, L-316, Livermore, CA 94551-0808

51. Donald M. Austin, 1100 Washington Ave. South, Room 101, University of Minnesota, Minneapolis, MN 55455

52. Owe Axelsson, Faculty of Mathematics and Informatics, University of Nijmegen, NL-6525 ED Nijmengen The Netherlands

53. Zhaojun Bai, Department of Mathematics, University of Kentucky, Lexington, KY 40506

54. Jesse Barlow, Dept. of Computer Science and Engineering, Pennsylvania State University, 220 Pond Laboratory, University Park, PA 16802-6106

55. Edward H. Barsis, Sandia National Laboratories, Computer Science and Mathematics, P.O. Box 5800, Albuquerque, NM 87185

56. Michael Berry, Department of Computer Science, 114 Ayres Hall, University of Tennessee, Knoxville, TN 37996-1301 
57. Rob Bisseling, University of Utrecht, Department of Mathematics, P.O. Box 80010, NL-3508 TA Utrecht, The Netherlands

58. Ake Bjorck, Dept. of Mathematics, Linkoping University, S-58183 Linkoping, Sweden

59. Petter Bjorstad, Institutt for Informatikk, Hoyteknologisenteret, N-5020 Bergen, Norway

60. Thierry Braconnier, 42 Ave G. Coriolis, 31057 Toulouse Cedex, France

61. Randall Bramley, Department of Computer Science, 215 Lindley Hall, Indiana University, IN 47405

62. Roger W. Brockett, Harvard University, 29 Oxford Street, Cambridge, MA 021382901

63. Peter Brown, Lawrence Livermore National Laboratory, P.O. Box 808, L-316, Livermore, CA 94551

64. Richard Brualdi, Dept. of Mathematics, 480 Lincoln Drive, Madison, WI 53706

65. Ralph Byers, 405 Snow Hall, Department of Mathematics, University of Kansas, Lawrence, KS 66045

66. Tony Chan, Dept. of Mathematics, University of California, Los Angeles, 405 Hilgard Avenue, Los Angeles, CA 90024-1555

67. Françoise Chaitin-Chatelin, 18 Rue Pierre Arnoux, 92190 Meudon, France

68. Paul Concus, Mathematics and Computing, Lawrence Berkeley Laboratory, 50A/2129, University of California, Berkeley, CA 94720

69. Jane Cullum, IBM T.J. Watson Research Center, P.O. Box 218, Yorktown Heights, NY 10598

70. Biswa Datta, Dept. of Mathematical Sciences, Northern Illinois University, DeKalb, IL 60115

71. Tim Davis, E301 CSE, University of Florida, Gainesville, FL 32611-2024

72. James Demmel, Dept. of Computer Science, University of California, 571 Evans Hall, Berkeley, CA 94720

73. John Dorning, Dept. of Nuclear Engineering Physics, Nuclear Reactor Facility, University of Virginia, Charlottesville, VA 22903-2442

74. Milo Dorr, Lawrence Livermore National Laboratory, P.O. Box 808, L-316, Livermore, CA 94551

75. Craig Douglas, 8 South Street, Cos Cob, CT 06807-1618

76. Tony Drummond, 42 Ave G. Coriolis, 31057 Toulouse Cedex, France

77. Donald J. Dudziak, Dept. of Nuclear Engineering, 110B Burlington Engineering Labs, North Carolina State University, Raleigh, NC 27695-7909

78. Iain Duff, Rutherford Appleton Laboratory, Didcot, Oxon 0X11OQX, England

79. Patricia Eberlein, Department of Computer Science, State University of New York, 226 Bell Hall, Buffalo, NY 14260 
80. Michael Eiermann, Institut für Angewandte Mathematik II, TU Bergakademie Freiberg, D-09596 Freiberg, Federal Republic of Germany

81. Stanley C. Eisenstat, Department of Computer Science, Yale University, P.O. Box 2158, New Haven, CT 06520

82. Tommy Elfving, University of Linkoping, MAI, 58183 Linkoping, Sweden

83. Howard C. Elman, Dept. of Computer Science, University of Maryland, College Park, MD 20742

84. Richard E. Ewing, Department of Mathematics, Texas A\&M University, College Station, TX 77843

85. Robert Falgout, Lawrence Livermore National Laboratory, P.O. Box 808, L-419, Livermore, CA 94551

86. Bernd Fischer, Universität Hamburg, Institut fur Angewandte Mathematik, Bundersstrasse 55, D-2000 Hamburg 13, Federal Republic of Germany

87. Geoffrey C. Fox, Northeast Parallel Architectures Center, 111 College Place, Syracuse University, Syracuse, NY 13244-4100

88. Valérie Frayssé, CERFACS, 42 Ave G. Coriolis, 31057 Toulouse Cedex, France

89. Roland Freund, AT\&T Bell Labs, Room 2C-420, 600 Mountain Avenue, Murray Hill, NJ 07974-0636

90. Robert Funderlic, Computer Science Department, Box 8206, 228 Withers Hall, North Carolina State University, Raleigh, NC 27695-8206

91. Walter Gander, Institut für Wissenschaftliches Rechnen, ETH-Zentrum, CH-8092 Zurich, Switzerland

92. C. William Gear, NEC Research Institute, 4 Independence Way, Princeton, NJ 08540-6621

93. J. Alan George, Vice President, Academic and Provost, Needles Hall, University of Waterloo, Waterloo, Ontario, Canada N2L 3G1

94. John Gilbert, Xerox Palo Alto Research Center, 3333 Coyote Hill Road, Palo Alto, CA 94304

95. Luc Giraud, 42 Ave G. Coriolis, 31057 Toulouse Cedex, France

96. Gene H. Golub, Dept. of Computer Science, Stanford University, Stanford, CA 94305

97. Nick Gould, Central Computing Department, Rutherford Appleton Laboratory, Chilton, Oxfordshire, 0X110QX, England

98. Serge Gratton, 42 Ave G. Coriolis, 31057 Toulouse Cedex, France

99. Joe Grcar, Sandia National Laboratories, Livermore, CA 94551-0969

100. Anne Greenbaum, Courant Institute, New York University, 251 Mercer Street, New York, NY 10012-1185

101. John Gustafson, Ames Laboratory, Iowa State University, Ames, IA 50011

102. Martin Gutknecht, IPS Supercomputing, ETH-Zentrum, CH-8092 Zurich, Switzerland 
103. Christian Halloy, Assistant Director of JICS, 104 South College, Joint Institute for Computational Science, University of Tennessee, Knoxville, TN 37996-1301

104. Sven. J. Hammarling, The Numerical Algorithms Group, Ltd., Wilkinson House, Jordan Road, Oxford OX2 8DR, United Kingdom

105. Michael T. Heath, National Center for Supercomputing Applications, 4157 Beckman Institute, University of Illinois, 405 N. Mathews Avenue, Urbana IL 618012300

106. Bruce Hendrickson, Sandia National Laboratories, Division 1422, Albuquerque, NM 87185

107. Nicholas Higham, Dept. of Mathematics, University of Manchester, 90 Half Edge Lane, Eccles, Manchester, M13 9PL, England

108. Alan Hindmarsh, Lawrence Livermore National Laboratory, P.O. Box 808, L-316, Livermore, CA 94551

109. Michael J. Holst, California Institute of Technology, Applied Mathematics 217-50, Pasadena, CA 91125

110. Fred Howes, Office of Scientific Computing, ER-7, Applied Mathematical Sciences, Office of Energy Research, Department of Energy, Washington, DC 20585

111. James M. Hyman, Los Alamos National Laboratory, MS B284, Group T-7, Los Alamos, NM 87545

112. Ilse Ipsen, Dept. of Mathematics, North Carolina State University, Raleigh, NC 27695-8205

113. Erxiong Jiang, Department of Mathematics, Fudan University, Shanghai 200433, People's Republic of China

114. Gary Johnson, Office of Scientific Computing, ER-7, Applied Mathematical Sciences, Office of Energy Research, Department of Energy, Washington, DC 20585

115. Bo Kågström, Institute of Information Processing, University of Umeå, S-901, 87 Umeå, Sweden

116. Malvyn H. Kalos, Cornell Theory Center, Engineering and Theory Center Bldg., Cornell University, Ithaca, NY 14853-3901

117. Hans Kaper, Mathematics and Computer Science Division, Argonne National Laboratory, 9700 South Cass Avenue, Bldg. 221, Argonne, IL 60439

118. Kenneth Kennedy, Dept. of Computer Science, Rice University, P.O. Box 1892, Houston, TX 77001

119. David Keyes, NASA Langley Research Center, ICASE, M/S L23C, Hampton, CA 23681-0001

120. David Kincaid, Center for Numerical Analysis, RLM Hall 13.150 , University of Texas at Austin, Austin, TX 78713-8510

121. Jacko Koster, 42 Ave G. Coriolis, 31057 Toulouse Cedex, France

122. Alan Laub, ECE Department, University of California at Santa Barbara, Santa Barbara, CA 93106-9560

123. Richard Lehoucq, Dept. of Computational and Applied Mathematics, P.O. Box 1892, Rice University, Houston, TX 77251-1892 
124. James E. Leiss, Rt. 2, Box 142C, Broadway, VA 22815

125. Steven J. Leon, Department of Mathematics, University of Massachusetts, Old Westport Road, North Dartmouth, MA 02747

126. John G. Lewis, Boeing Computer Services, P.O. Box 24346, M/S 7L-21, Seattle, WA 98124-0346

127. Thomas A. Manteuffel, Program in Applied Mathematics, Campus Box 526, University of Colorado, Boulder, CO 80309-0526

128. Osni Marques, 42 Ave G. Coriolis, 31057 Toulouse Cedex, France

129. Pontus Matstoms, University of Linkoping, MAI, 58183 Linkoping, Sweden

130. James McGraw, Lawrence Livermore National Laboratory, P.O. Box 808, M/S L-306 Livermore, CA 94550

131. Gerard Meurant, Centre d'Etudes de Limeil, Department MA, 94195 Villeneuve St. Georges, France

132. Juan Meza, Sandia National Laboratories, Division 8211, P.O. Box 969, Livermore, CA 94551-0969

133. Cleve Moler, The Mathworks, 24 Prime Park Way, Natick, MA 01760

134. Neville Moray, Dept. of Mechanical and Industrial Engineering, University of Illinois, 1206 West Green Street, Urbana, IL 61801

135. David Nelson, Director of Scientific Computing, ER-7, Applied Mathematical Sciences, Office of Energy Research, U.S. Dept. of Energy, Washington, DC 20585

136. Martin Ojangruen, 42 Ave G. Coriolis, 31057 Toulouse Cedex, France

137. Dianne P. O'Leary, Dept. of Computer Science, University of Maryland, College Park, MD 20742

138. Beth Ong, University of California at San Diego, Department of Mathematics, 9500 Gilman Drive, La Jolla, CA 92093-0112

139. James M. Ortega, Dept. of Applied Mathematics, Thornton Hall, University of Virginia, Charlottesville, VA 22901

140. Michael Overton, Courant Institute, New York University, 251 Mercer Street, New York, NY 10012-1185

141. Chris Paige, McGill University, School of Computer Science, McConnell Engineering Building, 3480 University Street, Montreal, Quebec Canada H3A 2A7

142. Beresford N. Parlett, Dept. of Mathematics, University of California, Berkeley, CA 94720

143. Joao Patricio, 42 Ave G. Coriolis, 31057 Toulouse Cedex, France

144. Linda R. Petzold, Dept. of Computer Science, University of Minnesota, 4-192 EE/CS Bldg, 200 Union Street S.E., Minneapolis, MN 55455-0159

145. Robert J. Plemmons, Dept. of Mathematics and Computer Science, Box 7311, Wake Forest University, Winston-Salem, NC 27109

146. Claude Pommerell, AT\&T Bell Laboratories, 2C-548A, Murray Hill, NJ 079740636 
147. James C. T. Pool, Deputy Director, Caltech Concurrent Supercomputing Facility, California Institute of Technology, Pasadena, CA 91125

148. Alex Pothen, Old Dominion University, Computer Science Department, Norfolk, VA 23529-0162

149. Chiara Puglisi, 42 Ave G. Coriolis, 31057 Toulouse Cedex, France

150. Bill Pulleyblank, IBM Research Division, P.O. Box 218, Yorktown Heights, NY 10598-0218

151. Padma Raghavan, Department of Computer Science, 657B Buehler Hall, University of Tennessee, Knoxville, TN 37996-1301

152. Lothar Reichel, Dept. of Mathematics, Kent State University, Kent, OH 44242

153. John Reid, Rutherford Appleton Laboratory, Oxon 0X11OQX, England

154. Werner C. Rheinboldt, Dept. of Mathematics and Statistics, University of Pittsburgh, Pittsburgh, PA 15260

155. Axel Ruhe, Department of Computer Science, Chalmers, S-41296 Göteborg, Sweden

156. Youcef Saad, Dept. of Computer Science, University of Minnesota, 4-192 EE/CS Bldg, 200 Union Street S.E., Minneapolis, MN 55455-0159

157. Faisal Saied, Department of Computer Science, 1304 W. Springfield Ave., University of Illinois at Urbana-Champaign, Urbana, IL 61801

158. Ahmed H. Sameh, Dept. of Computer Science, University of Minnesota, 4-192 EE/CS Bldg, 200 Union Street S.E., Minneapolis, MN 55455-0159

159. Michael Saunders, Systems Optimization Laboratory, Operations Research Dept., Stanford University, Stanford, CA 94305

160. Paul Saylor, Department of Computer Science, 1304 W. Springfield Ave., University of Illinois at Urbana-Champaign, Urbana, IL 61801

161. Hans Schneider, University of Wisconsin, Mathematics/Van Vleck Hall, 480 Lincoln Drive, Madison, WI 53706-1388

162. Robert Schreiber, RIACS, Mail Stop 230-5, NASA Ames Research Center, Moffet Field, CA 94035

163. Martin Schultz, Department of Computer Science, Yale University, P.O. Box 2158, Yale Station, New Haven, CT 06520

164. David S. Scott, Intel Scientific Computers, 15201 N.W. Greenbrier Parkway, Beaverton, OR 97006

165. Jeff Scroggs, Department of Mathematics, North Carolina State University, Box 8205 , Raleigh, NC 27695

166. John Shadid, Sandia National Laboratories, Parallel Computer Science Division, Division 1421, Albuquerque, NM 87185

167. Bob Skeel, Department of Computer Science, 1304 W. Springfield Ave., University of Illinois at Urbana-Champaign, Urbana, IL 61801

168. Danny Sorensen, Dept. of Mathematical Sciences, Rice University, P.O. Box 1892, Houston, TX 77251 
169. Gerhard Starke, Universität Karlsruhe, Institut fur Praktische Mathematik, Englerstrasse 2, D-76128 Karlsruhe, Federal Republic of Germany

170. G.W. Stewart, Dept. of Computer Science, University of Maryland, College Park, MD 20742

171. Zdenek Strakos, Czech Academy of Sciences, Pod vod. vezi 2, 18207 Praha 8, Czech Republic

172. Gilbert Strang, Massachusetts Institute of Technology, Room 2-240, Cambridge, MA 02319-4557

173. Ji-guang Sun, Institute for Information Processing, University of Umeå, S-901, 87 Umeå, Sweden

174. Xiobai Sun, Argonne National Laboratory, Bldg. 221, MCS Division, 9700 South Cass Ave., Argonne, IL 60439

175. Toshio Suzuki, Department of Mathematics, Yamanashi University, Kofu 400 , Japan

176. Paul N. Swartztrauber, National Center for Atmospheric Research, P.O. Box 3000, Boulder, CO 80307

177. Daniel Szyld, Dept. of Mathematics, Temple University, TU038-16, Philadelphia, PA 19122-2585

178. Philippe Toint, Department of Mathematics, Rue de Bruxelles 61, B-5000 Namur, Belgium

179. Vincent Toumazou, 42 Ave G. Coriolis, 31057 Toulouse Cedex, France

180. Anne Trefethen, Dept. of Computer Science, Cornell University, Upson Hall, Ithaca, NY 14853

181. Nick Trefethen, Dept. of Computer Science, Cornell University, Upson Hall, Ithaca, NY 14853

182. Raymond S. Tuminaro, Sandia National Laboratories, Mail Stop 1110, P.O. Box 5800 , Albuquerque, NM 87185

183. Henk van der Vorst, Dept. of Mathematics, Universiteit Utrecht, P.O. Box 80010, 3508 TA, Utrecht, The Netherlands

184. Paul Van Dooren, Universite Catholique de Louvain, Centre for Systems Engineering and Applied Mechanics (CESAME), Batiment Euler (Rm A.119), 4, avenue Georges Lemaitre, B-1348 Louvain-la-Neuve, Belgium

185. Sabine Van Huffel, Katholieke Universiteit Leuven, ESAT Laboratory, Kardinaal Mercierlaan 943001 Heverlee, Belgium

186. Charles Van Loan, Dept. of Computer Science, Cornell University, Ithaca, NY 14853

187. Jim Varah, Centre for Integrated Computer Systems Research, University of British Columbia, Office 2053-2324 Main Mall, Vancouver, British Columbia V6T 1W5, Canada

188. Richard S. Varga, Department of Mathematics, Kent State University, Kent, OH 44242 
189. Andy Wathen, School of Mathematics, University Walk, Bristol University, Bristol BS8 1TW, United Kingdom

190. Mary F. Wheeler, Rice University, Dept. of Mathematical Sciences, P.O. Box 1892, Houston, TX 77251

191. Andrew B. White, Computing Division, Los Alamos National Laboratory, P.O. Box 1663, MS-265, Los Alamos, NM 87545

192. David Young, University of Texas, Center for Numerical Analysis, RLM 13.150, Austin, TX 78731-8510

193. Hongyuan Zha, Department of Computer Science and Engineering, 220 Pond Laboratory, The Pennsylvania State University, University Park, PA 16802-6106

194. Zahari Zlatev, Hagens Alle 28, DK-2900 Hellerup, Copenhagen, Denmark

195. Office of Assistant Manager for Energy Research and Development, U.S. Dept. of Energy, Oak Ridge Operations Office, P.O. Box 2001 Oak Ridge, TN 37831-8600

196-197. Office of Scientific \& Technical Information, P.O. Box 62, Oak Ridge, TN 37830 